\title{
Is a Behavioral Graded Activity Program More Effective Than Manual Therapy in Patients With Subacute Neck Pain?
}

\author{
Results of a Randomized Clinical Trial
}

\author{
Jan J. M. Pool, PhD, PT, MT, ${ }^{*} \dagger$ Raymond W. J. G. Ostelo, PhD, PT, ${ }^{*} \ddagger$ Dirk L. Knol, PhD, ${ }^{*} \mathbb{S}$ \\ Johan W. S. Vlaeyen, PhD, I\| Lex M. Bouter, PhD, ${ }^{* * *}$ and Henrica C. W. de Vet, PhD*
}

Study Design. A randomized clinical trial.

Objective. To compare the effectiveness of a behavioral graded activity program with manual therapy in patients with subacute (4-12 weeks) nonspecific neck pain.

Summary of Background Data. Neck pain is a common complaint, for which many conservative therapies are available in primary care. There is strong evidence for manual therapy in combination with exercises. Psychosocial factors are also believed to play a role in chronic pain. The evidence of the effectiveness of a program focused on these factors is still unknown.

Methods. A randomized clinical trial was conducted, involving 146 patients with subacute nonspecific neck pain. The BGA program can be described as a time-contingent increase in activities from baseline toward predetermined goals. Manual therapy consists of specific spinal mobilization techniques and exercises. Primary outcomes were global perceived effect, the Numerical Rating Scale for pain and the Neck Disability Index. Secondary outcomes were the Tampa Scale for Kinesiophobia, the 4 Dimensional Symptom Questionnaire, and the Pain Coping and Cognition List. Measurements were carried out at baseline and $6,13,26$, and 52 weeks after randomization. Data are analyzed according to the intention-to-treat principle, using multilevel analysis.

Results. The success rates at 52 weeks, based on the GPE were $89.4 \%$ for the BGA program and $86.5 \%$ for MT. This difference was not statistically significant. For

From the *EMGO Institute for Health and Care research, VU University Medical Center, Amsterdam; †Medical Center Impact, Zoetermeer; łInstitute for Health Sciences, Faculty of Earth and Life Sciences VU University, Amsterdam; \$Department of Clinical Epidemiology and Biostatistics, VU University Medical Center, Amsterdam; IIDepartment of Medical, Clinical, and Experimental Psychology, Maastricht University, Maastricht, The Netherlands; |Department of Psychology, University of Leuven, Belgium; and *Executive Board of VU University, Amsterdam, The Netherlands.

Acknowledgment date: August 11, 2008. Revision date: July 21, 2009. Acceptance date: July 31, 2009.

The manuscript submitted does not contain information about medical device(s)/drug(s).

Netherlands Organization for Health Research and Development funds were received in support of this work. No benefits in any form have been or will be received from a commercial party related directly or indirectly to the subject of this manuscript.

Supported by Netherlands Organization for Health Research and Development (ZonMW) grant no-940-31-060.

The Medical Ethics Committee of the VU University Medical Center in Amsterdam approved the study protocol.

The authors declare no conflict of interest including financial interest and affiliations relevant to the manuscript.

Address correspondence and reprint requests to J. J. M. Pool, PhD, EMGO Institute for Health and Care research, VU University Medical Center, Van der Boechorststraat 7,1081 BT Amsterdam, The Netherlands; E-mail: jjm.pool@gmail.com or www.emgo.nl pain and disability, a difference was found in favor of the BGA program; mean difference for pain $=0.99(95 \% \mathrm{Cl}$ $0.15-1.83)$ and mean difference for NDI $=2.42(95 \% \mathrm{Cl}$ 0.52-4.32). All other differences between the interventions in the primary and secondary outcomes were not statistically significant.

Conclusion. Based on this trial it can be concluded that there are only marginal, but not clinically relevant, differences between a BGA program and MT.

Key words: neck pain, randomized clinical trial, behavioral graded activity, manual therapy. Spine 2010;35: 1017-1024

Neck pain is a common complaint. The point prevalence of neck pain in the general population of the Netherlands varies between $9 \%$ and $22 \%,{ }^{1,2}$ and approximately one third of all adults will experience neck pain during the course of 1 year. ${ }^{3}$ Some $5 \%$ to $10 \%$ of these patients will develop a chronic pain disorder. ${ }^{1}$ Once the neck pain becomes chronic (>12 weeks) $44 \%$ of the patients will consult their general practitioner (GP) in the following 12 months. ${ }^{1}$ There is no conclusive evidence regarding specific pathology in the majority of cases of acute or chronic neck pain ${ }^{4}$ so, consequently, most cases are labeled as nonspecific neck pain or neck pain of unknown origin. ${ }^{4}$ Psychological and social factors may aggravate and perpetuate neck pain. ${ }^{5,6}$

The therapeutic methods that are most frequently used for the treatment of neck pain are exercises, manipulative therapies, mobilization, massage, physical methods, and multidisciplinary biopsychosocial rehabilitation. ${ }^{7}$ Although the evidence regarding the effectiveness of most conservative types of therapy for neck pain is still inconclusive, the updated Cochrane review carried out by Gross et $\mathrm{al}^{8}$ concluded that there was strong evidence for manipulation and/or mobilization, if combined with exercises. Furthermore, the results of a recent randomized clinical trial (RCT) carried out by Hoving et $^{9 l^{9-11}}$ in patients with subacute and chronic neck pain showed a significant difference in effectiveness and cost-effectiveness in favor of MT, compared with physical therapy or usual care from the GP, both in the short and the long-term follow-up.

Psychological and social factors are believed to play a role in the transition from acute to chronic pain and disability. ${ }^{6,12,13}$ Consequently, the emphasis in treating patients with subacute and chronic pain is increasingly 
on behavioral treatment, based on operant, cognitive, or respondent techniques. ${ }^{13-16}$ Behavioral treatment focuses on reducing disability through the modification of environmental contingencies and cognitive processes. Especially for patients who interpret pain as threatening (pain catastrophyzing) and are afraid that movement might be harmful (kinesiophobia), behavioral treatment seems to be effective. ${ }^{13,17}$ This led to the development of a behavioral graded activity (BGA) program. The evidence of the effectiveness of a BGA program, which is still emerging, is mainly from studies on low back pain. ${ }^{18-20}$ However, the effectiveness of a BGA program for neck pain is still unknown. We hypothesize that psychological factors such as those described above, will influence neck pain, and we suggest that a BGA program will be at least as effective as MT for patients with subacute neck pain. ${ }^{21}$

\section{- Materials and Methods}

The methods have been described elsewhere in detail. ${ }^{21}$

\section{Selection of Patients}

The study aims to include 90 patients per treatment arm. Although arbitrary this is based on the expectation that in the manual therapy group $70 \%$ of the patients will recover. To detect a difference of $20 \%$ between the 2 treatment groups, which is considered as clinically important, 84 patients are required for each treatment group. This calculation is based on the dichotomised primary outcome measure "perceived recovery," defined as the percentage of patients who are reported to have recovered. The sample size calculation concerns an $\alpha$ of 0.05 and a power $(1-\beta)$ of $90 \%$.

Subacute neck pain was defined as pain in the cervical region, existing for at least 4 weeks, but no longer than 12 weeks. The neck pain could radiate to the shoulder region or the upper extremities and/or be accompanied by headaches, but the main complaint must concern the neck. The other inclusion criteria were age between 18 and 70 years, and a new episode of nonspecific neck pain, defined as no neck pain in the previous 4 months. The exclusion criterion was specific neck pain, for example because of rheumatoid arthritis, disc herniation, neurologic diseases, or malignancy. Patients with whiplashassociated disorders were included, unless they had an unsettled insurance claim during the intake period. During the first GP consultation, these criteria were assessed and the patient was informed about the study. Eligible patients who were interested in participation were referred to the research assistant, who informed them about the consequences of participation and rechecked the inclusion criteria. Patients who were eligible and agreed to participate were asked to sign the informed consent form before the baseline measurement was performed. The baseline measurement consisted of collecting data on demographic variables, potential prognostic factors, and outcome measurements.

The Medical Ethics Committee of the VU University Medical Center in Amsterdam approved the study protocol.

\section{Randomization Procedure}

After the baseline measurement the patients were randomly assigned either to the BGA program or to MT. The treatment allocation was concealed, trough the use of numbered, opaque, and sealed envelopes, based on a computer-generated list, and prepared by an independent person before the start of the inclusion period.

\section{Blinding}

The research assistant who was responsible for all measurements, and visited the patient at home, was blinded for the treatment allocation. The patients were aware of the treatment they received.

\section{Behavioral Graded Activity Program}

The physical therapists (PTs) who provided the BGA program in this trial had followed additional courses in the biopsychosocial approach of pain problems, to make sure they had the necessary skills. Furthermore, all the PTs had more than 10 years of clinical experience. For this study they attended an additional a 2-day training course, which consisted of a theoretical part, in which the principles of a BGA program were discussed, and a practical part, in which skills were trained using case reports. The course supervised by an experienced behavioral therapist (A.K.) and a psychologist (J.W.S.V.). The BGA program was based on time-contingent management, as described in more detail by Fordyce 22,23 and applied by Lindstrom and Ostelo. ${ }^{15,24}$ The emphasis of the treatment is on operant conditioning.

Core elements of a BGA program are: (1) decrease in pain behavior and increase in "healthy" behavior, (2) improvement of function and no focus on pain reduction, (3) the patient is responsible for the treatment and has an active role, and (4) the therapist acts as a coach. The therapy is based on a typical "hands-off" approach.

From the baseline level, and working toward preset goals, the patients were trained to follow a gradually increasing exercise program, which consisted of a maximum of 18 sessions of approximately 30 minutes.

\section{Manual Therapy Treatment}

Manual therapists provided the MT. In the Netherlands after completing their training, PTs follow a 3-year postgraduate course in manipulation and specific mobilization techniques to become certified and registered as an MT by the Royal Dutch Society for Physical Therapy (KNGF). The aims of the MT treatment are: (1) to restore restricted movement, stimulating natural recovery and adaptive processes in relation to the functionality of movement, ${ }^{21}(2)$ to reduce pain, (3) to increase the patient's level of activities and participation, and (4) to prevent recurrences. The treatment consists of manipulation and specific mobilization techniques. It is also standard practice to give additional exercises and advice during MT treatment. The therapists were allowed to provide a maximum of 6 treatment sessions, each with a duration of 30 to 45 minutes, within 6 weeks. The MT was similar to the MT provided in the trial by Hoving et al. ${ }^{9}$

A detailed description of both interventions has been published elsewhere. ${ }^{21}$

\section{Outcome Measures}

The baseline measurement consisted of data on patient characteristics, demographic variables, potential prognostic factors, and outcome measurements (Table 1). Furthermore, the patients completed questionnaires at $6,13,26$, and 52 weeks after randomization. The primary outcome measurements were: (1) Global Perceived Effect (GPE) $)^{25,26}$ measured on a 7-point scale ranging from "completely recovered" to "worse than ever" (recovery was a priori defined as "completely recovered" or 
Table 1. Baseline Characteristics of the 2 Treatment Groups

\begin{tabular}{|c|c|c|}
\hline Variable & $\begin{array}{l}\text { BGA Programme } \\
\qquad(\mathrm{n}=71)\end{array}$ & $\begin{array}{l}\text { Manual Therapy } \\
\quad(\mathrm{n}=75)\end{array}$ \\
\hline Age (mean; SD) & $44.5(12.0)$ & $45.6(11.1)$ \\
\hline Gender ( $\%$ female) & 59 & 63 \\
\hline \multicolumn{3}{|l|}{ Complaints } \\
\hline Pain (\%) & 93 & 88 \\
\hline Limitation of movement (\%) & 58 & 56 \\
\hline Headache $(\%)$ & 63 & 69 \\
\hline Dizziness (\%) & 37 & 28 \\
\hline Fatigue (\%) & 56 & 48 \\
\hline \multicolumn{3}{|l|}{ Pain intensity (NRS 0-10) } \\
\hline Current pain (mean, SD) & $5.5(2.2)$ & $5.0(2.1)$ \\
\hline $\begin{array}{l}\text { Mean pain previous month } \\
\text { (mean, SD) }\end{array}$ & $6.2(1.5)$ & $6.0(1.5)$ \\
\hline NDI (mean, SD) & $14.7(6.2)$ & $13.4(7.4)$ \\
\hline \multicolumn{3}{|l|}{ Graded chronic pain scale (\%) } \\
\hline Low intensity & 11 & 15 \\
\hline High intensity & 58 & 59 \\
\hline Moderate limiting & 15 & 12 \\
\hline Severe limiting & 16 & 15 \\
\hline \multicolumn{3}{|l|}{ SF 36 (mean, SD) } \\
\hline Physical component summary & $42.5(7.9)$ & $44.8(7.3)$ \\
\hline Mental component summary & $46.8(11.4)$ & $47.5(12.2)$ \\
\hline \multicolumn{3}{|c|}{$\begin{array}{l}\text { The baseline characteristics of the Pain Coping and Cognition List, the } 4 \\
\text { Dimensional Symptom Questionnaire and the Tampa Scale for Kinesiophobia } \\
\text { can be found in Table 3. } \\
\text { BGA indicates behavioral graded activity; NRS, Numerical Rating Scale; NDI, } \\
\text { Neck Disability Index; SF-36, Short Form-36; SD, standard deviation. }\end{array}$} \\
\hline
\end{tabular}

"much improved," as reported by the patient); (2) the severity of current neck pain, scored on an 11-point Numerical Rating Scale (NRS) ranging from 0 "no pain" to 10 "very severe pain"; and (3) the neck-specific functional status measured with the 10-item Neck Disability Index. ${ }^{27}$

The secondary outcomes were (1) fear of movement, assessed with the Tampa Scale for Kinesiophobia $\left(\right.$ TSK) ${ }^{28}$; (2) pain catastrophysing, pain coping, and pain control (external and internal control) based on the Pain Coping and Cognition List (PCCL) ${ }^{29}$; (3) distress, depression, fear, and somatisation, assessed with the 4 Dimensional Symptom Questionnaire $(4 \mathrm{DSQ})^{30} ;(4)$ the intensity of pain, interference with activities, and persistence of pain, assessed according to the Graded
Chronic Pain Scale (GCPS) ${ }^{31,32}$; and (5) general health status, evaluated with the Short Form 36 (SF-36). ${ }^{33}$

\section{Statistical Analyses}

The statistical analyses were performed according to the intention-to-treat principle. To determine the effectiveness of the interventions over the follow-up period, multilevel analyses were performed. In the analyses, patients clustered under therapists and repeated measurement clustered within a patient, were taken into account. Therefore, we used linear multilevel analyses for continuous variables, such as the NDI and the NRS, and logistic multilevel analyses for dichotomous variables, such as the GPE. We included the following levels: repeated measures (time), patient, and therapist. The analyses took baseline scores into account and focused on the interaction between repeated measurement and intervention. The resulting regression coefficients (continuous variables) or odds ratios (dichotomous variables) can be interpreted as the difference in patient outcomes between the 2 groups at a certain follow-up moment. Per protocol analyses were also performed, excluding all patients with deviations from the protocol. For the BGA program a deviation of the protocol was defined as: not conducted the baseline level in the initial phase of the treatment, or suspension of therapy, applying "hands-on" therapy, or using a pain-contingent approach. ${ }^{21}$ For the MT a deviation of the protocol was defined as not using manipulation or specific mobilization techniques.

To calculate the sum scores, occasional missing items within a questionnaire $(<5 \%)$ were imputed, using a SPSS syntax for missing value imputation. ${ }^{34}$ When applying multilevel analyses to longitudinal data, no imputation strategy for missing questionnaires is necessary. It has been demonstrated that multilevel analyses are very flexible in handling these missing questionnaires. ${ }^{35}$ The analyses were performed in SPSS 12.0 and MLwiN 2.02.

\section{Results}

\section{Participants}

From January 2003 until January 2005 a total of 163 patients were referred to the research assistant. One hundred forty-six patients met all the inclusion criteria and

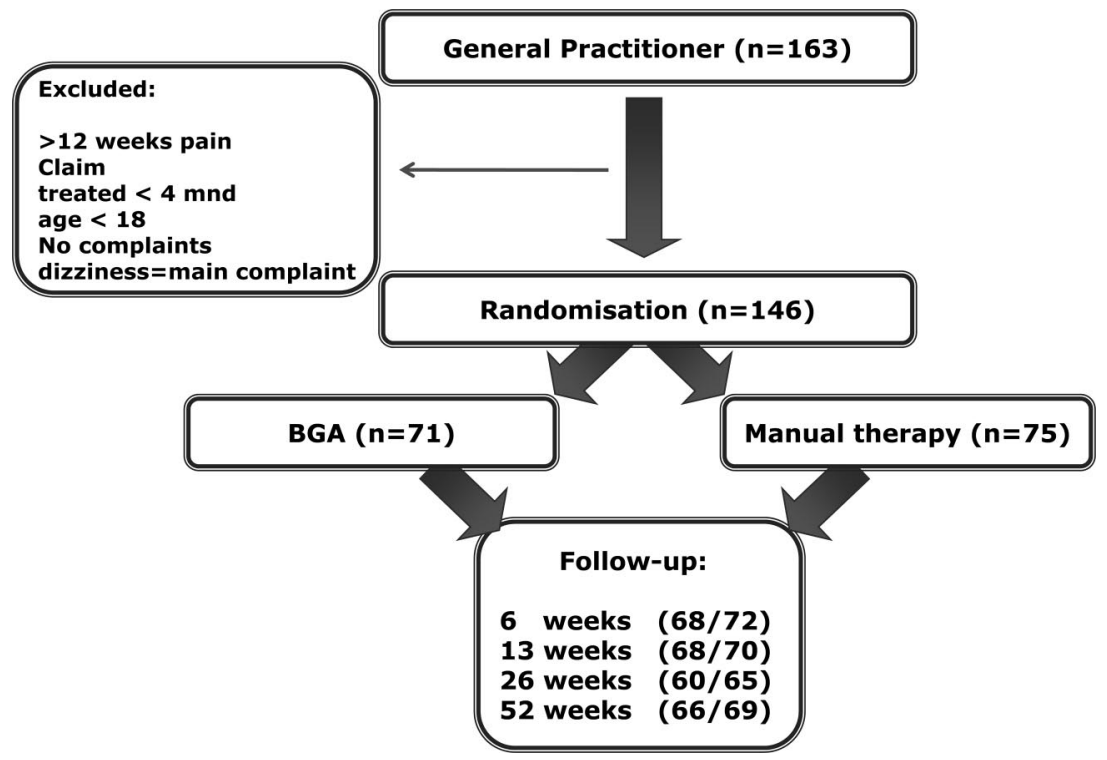


Table 2. Multilevel Model-Based Mean Scores at Baseline, Posttreatment and Follow-up and the Odds Ratios and Regression Coefficients for the Primary Outcome Measures

\begin{tabular}{|c|c|c|c|}
\hline Primary Outcome Measure & BGA Programme & Manual Therapy & Odds Ratio $(95 \% \mathrm{Cl})$ \\
\hline \multicolumn{4}{|c|}{ GPE $(\% \text { yes })^{*}$} \\
\hline \multicolumn{4}{|c|}{ Overall effect: $\chi^{2}=5.26 ; 4 d f(P=0.26)$} \\
\hline $13 \mathrm{wk}$ & $86 \%$ & $70 \%$ & 0.39 (0.12 to 1.28$)$ \\
\hline $52 \mathrm{wk}$ & $89 \%$ & $87 \%$ & $\begin{array}{c}0.76(0.21 \text { to } 2.68) \\
\text { Regression } \\
\text { Coefficient }(95 \% \mathrm{CI})\end{array}$ \\
\hline \multicolumn{4}{|c|}{ Current pain (NRS range $0-10$ ) } \\
\hline \multicolumn{4}{|c|}{ Overall effect: $\chi^{2}=5.26 ; 4 d f(P=0.23)$} \\
\hline Baseline & 5.47 & 5.13 & \\
\hline 13 wk & 1.83 & 2.15 & $0.66(-0.18$ to 1.49$)$ \\
\hline $52 \mathrm{wk}$ & 1.03 & 1.68 & $0.99(0.15$ to 1.83$)$ \\
\hline \multicolumn{4}{|c|}{ NDI (range 0-50) } \\
\hline \multicolumn{4}{|c|}{ Overall effect: $\chi^{2}=9.66 ; 4 d f(P=0.05)$} \\
\hline Baseline & 14.68 & 13.40 & \\
\hline 13 wk & 5.55 & 6.28 & 2.05 (0.17 to 3.93$)$ \\
\hline $52 \mathrm{wk}$ & 4.28 & 5.42 & 2.42 (0.52 to 4.32 ) \\
\hline
\end{tabular}

signed the informed consent form. Figure 1 presents a flow chart of the study. The baseline characteristics of the patients in the two groups were very similar (Table 1).

\section{Effect of the Interventions}

Intention-to-Treat Analyses. Table 2 shows the percentages and odds ratios for the GPE, and the means and regression coefficients (that can be interpreted as mean difference) for the NRS and the NDI at 13 and 52 weeks. At 52 weeks, the BGA group scored slightly better, for the GPE expressed as an OR of 0.76 (0.21-2.68), for the NRS expressed as a regression coefficient or mean difference of $0.99(0.15-1.83)$ points, and for the NDI expressed as a mean difference of $2.42(0.52-4.32)$ points (Figures 2-4). The only statistically significant overall effect was found on the NDI in favor of the BGA treatment. This effect was present at all follow-up moments. For all the other primary outcomes there was no statistically significant difference between the two groups.
Considering the results at 13 weeks, the effects of the BGA treatment were achieved earlier than the effects of the MT.

Table 3 shows the effects of the two interventions on the secondary outcome measurements, the PCCL, the 4 DSQ, and the TSK. There was no statistically significant overall difference in effect between the two interventions. Only somatisation, a domain within the 4 DSQ, showed a significant difference in favor of the BGA treatment at 52 weeks. There were also no differences in effects between the secondary outcomes SF 36 and GCPS (data not shown here).

Per Protocol Analyses. Despite the training course and the follow-up sessions, we found that only $52.1 \%$ of the PTs adhered adequately to the BGA program, according to the strict principles. On the other hand, $80 \%$ the manual therapy treatment sessions consisted of manipulations and/or mobilizations with or without exercises. The results of the
Figure 2. Results of the Global Perceived Effect during the 1-year follow-up.

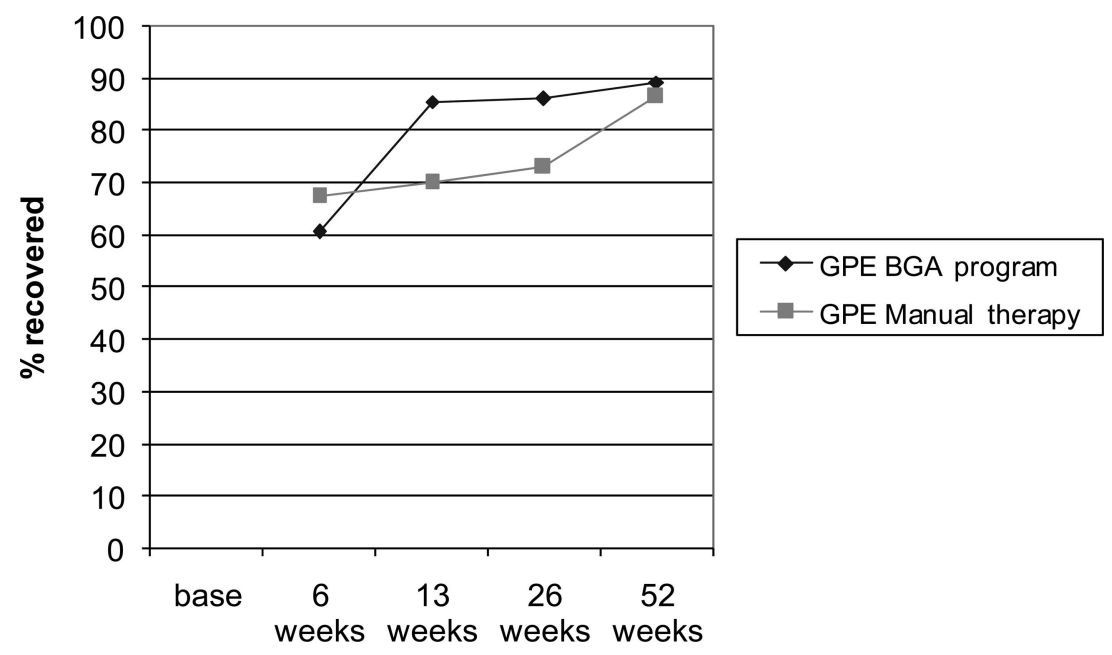


Figure 3. Results of mean pain intensity on the Numerical Rating Scale during the 1-year follow-up.

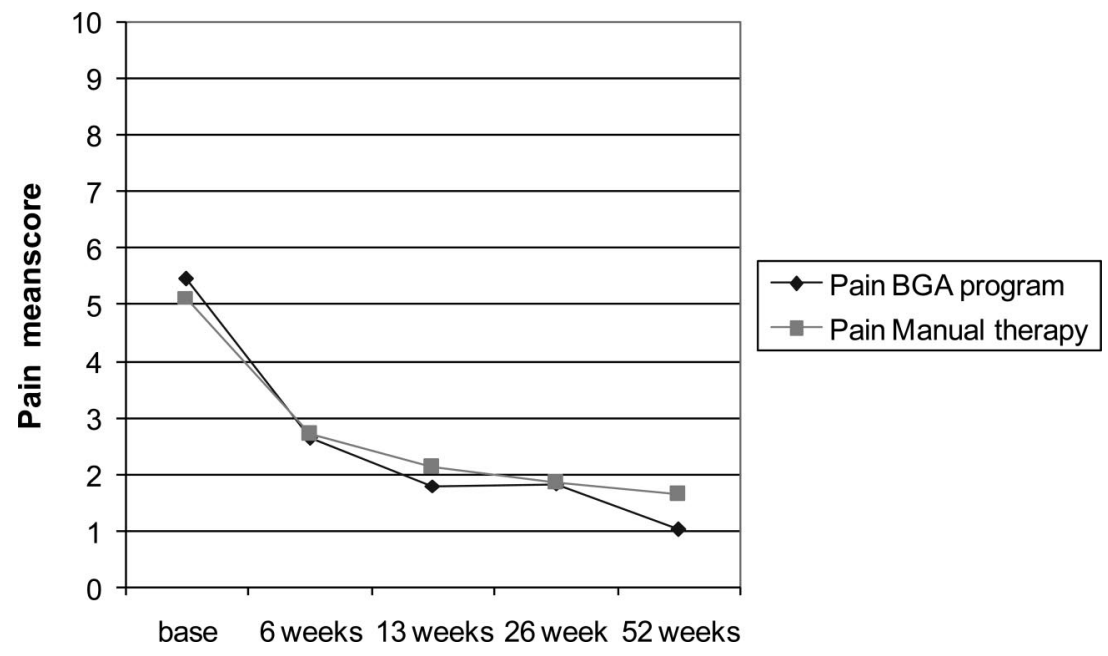

per protocol analyses (data not shown) were very similar to the results of the intention-to-treat analyses.

\section{Health Care Utilization}

The MT uses a mean of 5.2 treatments versus 8.2 treatments in BGA program. Over the same period, the mean number of visits to a general practitioner $(\mathrm{GP})$ was 0.5 in MT versus 0.8 in the BGA program. Also, the mean number of patients visiting a specialist (neurosurgeon, neurologist, or orthopaedic surgeon) was 0.05 in MT versus 0.03 in the BGA program. The differences were not statistically significant.

For indirect costs the mean number of days for absenteeism paid labor was 2.1 in MT versus 4.3 in the BGA program.

\section{Discussion}

We compared the effectiveness of a BGA program to MT for the treatment of subacute neck pain. In previous studies MT has been found to be the most appropriate therapy. It was concluded that on the primary outcome measures there was a marginal difference of effect in favor of the BGA treatment, which only reached statistical significance on the NDI. Moreover, the effect of a BGA treatment was achieved earlier than the effect of the MT. We found no significant difference in effect on the secondary outcomes, which included mainly psychological measures.

The minimal clinically important change has been estimated to be at least 3.5 points on the NDI, and at least 2.5 points on the NRS. ${ }^{36}$ In the current trial the change scores on the NDI were 10.4 points for the BGA group and 8.0 points for the MT group. On the NRS these mean changes were 4.4 and 3.5 , respectively. This implies that in both intervention groups substantial improvements were observed, but that the differences in improvements between the two groups were small. It can be questioned to what extent the improvements in the two intervention groups are because of natural recovery from subacute neck pain. In a previous trial carried out by Hoving et al, MT appeared to be clearly more effective than usual care (mainly based on a "wait and see" policy) provided by GPs for patients with subacute and chronic neck pain. Therefore, we conclude that both interventions are effective, and that the natural course is not fully responsible for the improvement achieved in the two interventions.

Because of the focus on improving functional status and a lack of focus on pain reduction, a significant difference in functional status (NDI) was in line with the expectations. Surprisingly, no differences in effects were

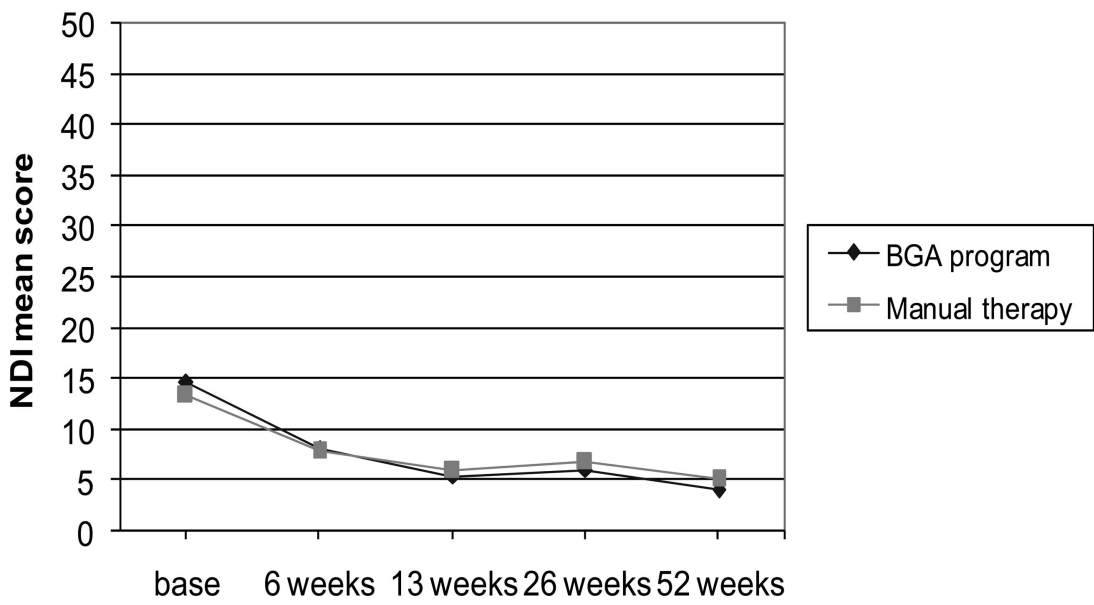

Figure 4. Results of the Neck Disability Index during the 1-year follow-up. 
Table 3. Multilevel Model-Based Mean Scores at Baseline, Posttreatment and Follow-up and the Regression Coefficients for the Secondary Outcome Measures

\begin{tabular}{|c|c|c|c|}
\hline Scales of PCCL & BGA Programme & Manual Therapy & Regression Coefficients $(95 \% \mathrm{CI})$ \\
\hline \multicolumn{4}{|c|}{ Catastrophysing (range 1-6) } \\
\hline \multicolumn{4}{|c|}{ Overall effect: $\chi^{2}=2.53 ; 4 d f(P=0.64)$} \\
\hline Baseline & 2.25 & 2.35 & \\
\hline 13 wk & 1.72 & 1.77 & $-0.04(-0.27$ to 0.19$)$ \\
\hline $52 \mathrm{wk}$ & 1.62 & 1.78 & $0.06(-0.17$ to 0.30$)$ \\
\hline \multicolumn{4}{|c|}{ Coping (range 1-6) } \\
\hline \multicolumn{4}{|c|}{ Overall effect: $\chi^{2}=5.00 ; 4 d f(P=0.29)$} \\
\hline Baseline & 3.30 & 3.46 & \\
\hline 13 wk & 3.34 & 3.41 & $-0.08(-0.36$ to 0.19$)$ \\
\hline 52 wk & 3.54 & 3.42 & $-0.28(-0.56$ to 0.00$)$ \\
\hline \multicolumn{4}{|c|}{ Internal pain control (range 1-6) } \\
\hline \multicolumn{4}{|c|}{ Overall effect: $\chi^{2}=6.03 ; 4 d f(P=0.20)$} \\
\hline Baseline & 3.67 & 3.83 & \\
\hline 13 wk & 4.10 & 3.97 & $-0.28(-0.54$ to -0.03$)$ \\
\hline 52 wk & 4.13 & 4.11 & $-0.18(-0.44$ to 0.08$)$ \\
\hline \multicolumn{4}{|c|}{ External pain control (range 1-6) } \\
\hline \multicolumn{4}{|c|}{ Overall effect: $\chi^{2}=5.78 ; 4 d f(P=0.22)$} \\
\hline Baseline & 2.94 & 3.21 & \\
\hline $13 \mathrm{wk}$ & 2.27 & 2.73 & $0.19(-0.07$ to 0.45$)$ \\
\hline 52 wk & 2.44 & 2.60 & $-0.11(-0.37$ to 0.16$)$ \\
\hline
\end{tabular}

In all multilevel analyses the baseline scores were accounted for. Regression coefficients can be interpreted as mean differences between interventions. BGA indicates behavioural graded activity; PCCL, Pain Coping and Cognition List; $\mathrm{Cl}$, confidence interval; $d f$, degrees of freedom.

found on kinesiophobia, coping styles, or other psychological variables. There might be several explanations why the BGA treatment was not more convincingly beneficial than MT, as we had anticipated:

1. One reason could be that the hypothesized working mechanism of a BGA program was not effective for patients with subacute neck pain. Improvement in psychological outcomes such as fear of movement, distress, coping, depression, etc., was to be expected, thereby resulting in an increase level of function. However, after the intervention there was no significant change in these psychological variables. This could be because of the mainly low initial scores for these psychological outcomes at baseline: very few patients scored "moderate" to "high" on the 4 DSQ, PCCL or TSK, so no great improvement could be expected.

2. Another explanation might concern the attitudes and beliefs of the PTs who provided the BGA treatment. The training course for the PTs before the intervention was aimed, among other things, at changing their attitude from a biomedical attitude to a biopsychosocial attitude. We regard this biopsychosocial attitude as vital for the optimal provision of a BGA treatment. Because the strict protocol of the BGA program, which was based on a hands-off strategy, was not always adequately adhered to, we question the presence of a biopsychosocial attitude in PTs, even if they had already followed previous courses. The principles of BGA are not easily learned or applied, as was also obvious in GP care. ${ }^{37,38}$ A 2-day training course is perhaps insufficient for the PTs to adopt a sufficiently strong biopsychosocial attitude, and this may have hampered optimal provision of the therapy.
3. Yet another explanation might concern insufficient flexibility of daily practice in primary care. A BGA program requires a rather strict protocol in terms of organization and frequency of treatment sessions. It turned out to be difficult to adhere to a strict protocol, and this might have resulted in a sub optimal provision of the BGA treatment in this trial.

4. Another factor, which can influence the effectiveness of BGA treatment on neck pain, is the duration of the complaints. In this trial we included patients with subacute neck pain. Consequently, many patients had a quick recovery, so the predetermined treatment aims were achieved sooner than was expected. As a consequence, the intensity of the program, as determined at baseline, was too low for some patients, and therefore compliance with the strict protocol was also rather low.

Another remark can be made concerns the difference in treatment frequency and the difference in duration of treatment time between MT and the BGA program. It can be questioned if the time factor in favor of BGA is a considerable factor which could influence the outcome of the trial. However, as this was a pragmatic design we were not primarily interested in the specific components of a treatment but in the effect of the treatment as a whole.

Although we initially aimed to include 180 participants we were only able to include 146 patients. The GPs were responsible for the recruitment of patients, but despite the fact that each of the participating GPs $(n=72)$ who were contacted individually by the researcher agreed to participate, only $49 \%$ actually referred patients to the study. There was also a change in legislation during the recruitment period which delisted physical 
therapy from basic health care insurance, and from January 2005 onwards patients needed an additional health care insurance to cover the costs of physical therapy and MT. GPs were therefore more reluctant than before to refer patients to a physical therapist or a manual therapist. Considering the marginal differences in effectiveness between the interventions, it seems unlikely that there would have been any drastic change in results with the inclusion of an additional 34 patients.

We therefore consider that the number of patients included in this study was sufficient to draw conclusions.

In the trial by Hoving et al, ${ }^{9} \mathrm{MT}$ appeared to be more effective than usual care or physical therapy for patients with subacute and chronic neck pain, but in our study the BGA treatment appeared to be slightly more effective than MT. Based on the results of both studies, we conclude that patients with subacute nonspecific neck pain can benefit from both interventions. This information regarding the evidence of both interventions should be discussed with patient who are about to be treated for their subacute neck pain. In this way patient preferences, in addition to the evidence, can be taken into account when deciding what treatment the patient should receive.

In future studies that aim to study the effect of a BGA program, it seems reasonable to suggest that the focus should be more on patients with psychological characteristics, such as high scores for fear of movement, distress, depression, etc. In the current study we did not select patients with psychological characteristics, however, these patients might benefit most from a BGA program.

\section{- Key Points}

- In a randomized clinical trial that included 146 patients with subacute neck pain, the effectiveness of a behavioral graded activity program was assessed.

- Little is known about the effectiveness of a behavioral graded activity program in patients with subacute neck pain and no randomized controlled trials exist that assess a structural behavioral treatment in patients with subacute neck pain.

- After 1 year of follow-up, there were no statistically significant or clinically relevant differences between a behavioral graded activity program and manual therapy as provided by physiotherapists for patients with subacute neck pain.

- Baseline scores on psychosocial questionnaires in subacute neck pain patients are low to moderate.

\section{Acknowledgments}

The authors thank Dr. Annelies Pool-Goudzwaard for editing the manuscript. They also thank the participating manual therapists, physical therapists, general practitioners, and patients.

\section{References}

1. Borghouts JA, Koes BW, Vondeling H, et al. Cost-of-illness of neck pain in The Netherlands in 1996. Pain 1999;80:629-36.
2. Picavet HSJ, Schouten JSAG. Musculoskeletal pain in the Netherlands: prevalences, consequences and risk groups, the DMC3-study. Pain 2003; 102:167-78.

3. Croft PR, Lewis M, Papageorgiou AC, et al. Risk factors for neck pain: a longitudinal study in the general population. Pain 2001;93:317-25.

4. Bogduk N, Barnsley L. Back pain and neck pain: an evidence-based update. In: Devor M, Rowbotham MC, Wiesenfeld-Hallin Z, eds. Progress in Pain Research and Management, 16 ed. Seattle: IASP Press; 2000;371-7.

5. Ariens GAM, Borghouts AJ, Koes BW. Neck pain. In: Crombie IK, ed. Epidemiology of Pain. Seattle: IASP Press; 1999;235-55.

6. Linton SJ. A review of psychological risk factors in back and neck pain. Spine 2000;25:1148-56.

7. Pool JJM, Rubinstein SM, van Tulder MW. The current evidence for conservative treatment modalities for acute and chronic neck pain. A summary of findings. Manuelle Medizin 2005;43:297-304.

8. Gross AR, Hoving JL, Haines TA, et al. A Cochrane review of manipulation and mobilization for mechanical neck disorders. Spine 2004;29:1541-8.

9. Hoving JL, Koes BW, de Vet HC, et al. Manual therapy, physical therapy, or continued care by a general practitioner for patients with neck pain. A randomized, controlled trial. Ann Intern Med 2002;136:713-22.

10. Hoving JL, de Vet HC, Koes BW, et al. Manual therapy, physical therapy, or continued care by the general practitioner for patients with neck pain: longterm results from a pragmatic randomized clinical trial. Clin J Pain 2006;22: $370-7$.

11. Korthals-de Bos IB, Hoving JL, van Tulder MW, et al. Cost effectiveness of physiotherapy, manual therapy, and general practitioner care for neck pain: economic evaluation alongside a randomised controlled trial. BMJ 2003; 326:911.

12. Gatchel RJ. Psychological disorders and chronic pain. Cause-and effect relationships. In: Gatchel RJ, Turk DC, eds. Psychological Approaches to Pain Management. A Practitioners's Handbook. New York: The Guilford Press; 1996;33-52.

13. Vlaeyen JW, Linton SJ. Fear-avoidance and its consequences in chronic musculoskeletal pain: a state of the art. Pain 2000;85:317-32.

14. Turner JA, Keefe FJ. Cognitive-behavioural therapy for chronic pain. Devor M, Rowbotham MC, Wiesenfeld-Hallin Z. Progress in Pain Research and Management. Seattle: IASP Press;1999;523-33.

15. Ostelo RW, Koke AJ, Beurskens AJ, et al. Behavioral-graded activity compared with usual care after first-time disk surgery: considerations of the design of a randomized clinical trial. J Manipulative Physiol Ther 2000;23: 312-9.

16. van Tulder MW, Goossens ME, Hoving JL. Nonsurgical treatment of chronic neck pain. In: Alf Nachemson, Egon Jonsson, eds. Neck and Back Pain: The Scientific Evidence of Causes, Diagnosis, and Treatment. Philidelphia: Lippincott Williams \& Wilkins; 2000;339-54.

17. Nederhand MJ, IJzerman MJ, Hermens HJ, et al. Predictive value of fear avoidance in developing chronic neck pain disability: consequences for clinical decision making. Arch Phys Med Rehabil 2004;85:496-501.

18. Ostelo RW, de Vet HC, Vlaeyen JW, et al. Behavioral graded activity following first-time lumbar disc surgery: 1-year results of a randomized clinical trial. Spine 2004;28:1757-65.

19. Bastiaenen CH, de Bie RA, Wolters PM, et al. Effectiveness of a tailormade intervention for pregnancy-related pelvic girdle and/or low back pain after delivery: short-term results of a randomized clinical trial [ISRCTN08477490]. BMC Musculoskelet Disord 2006;7:19.

20. Staal JB, Hlobil H, Twisk JW, et al. Graded activity for low back pain in occupational health care: a randomized, controlled trial. Ann Intern Med 2004;140:77-84.

21. Pool JJ, Ostelo RW, Koke AJ, et al. Comparison of the effectiveness of a behavioural graded activity program and manual therapy in patients with sub-acute neck pain: design of a randomized clinical trial. Man Ther 2006; 11:297-305.

22. Fordyce WE. Behavioral Methods for Chronic Pain and Illness. St. Louis: Mosby; 1976.

23. Fordyce WE, Fowler RS Jr, Lehmann JF, et al. Operant conditioning in the treatment of chronic pain. Arch Phys Med Rehabil 1973;54:399-408.

24. Lindstrom I, Ohlund C, Eek C, et al. The effect of graded activity on patients with subacute low back pain: a randomized prospective clinical study with an operant-conditioning behavioral approach. Phys Ther 1992;72:279-90.

25. Feinstein AR. Clinimetrics, 1 st ed. New Haven and London: Yale University Press; 1987;91-103.

26. Beurskens AJ, de Vet HC, Koke AJ. Responsiveness of functional status in low back pain: a comparison of different instruments. Pain 1996;65: $71-6$.

27. Vernon H, Mior S. The Neck Disability Index: a study of reliability and validity. J Manipulative Physiol Ther 1991;14:409-15. 
28. Kori SH, Miller RP, Todd DD. Kinisophobia: a new view of chronic pain behavior. Pain Management 1990;35-43.

29. Stomp-van der Berg SGM, Vlaeyen JW, ter Kuile MM, et al. Meetinstrumenten chronische pijn deel 2 Pijn Coping en Cognitie Lijst (PCCL). Maastricht: Pijn Kennis Centrum Academisch Ziekenhuis Maastricht; 2001.

30. Terluin B, van Marwijk HW, Ader HJ et al. The Four-Dimensional Symptom Questionnaire (4DSQ): a validation study of a multidimensional self-report questionnaire to assess distress, depression, anxiety and somatization. $B M C$ Psychiatry 2006;6:34.

31. Von Korff MR, Ormel J, Keefe FJ, et al. Grading the severity of chronic pain. Pain 1992;50:133-49.

32. Von Korff MR. Epidemiologic and survey methods: chronic pain assessment. In: Turk DC, Melzack R, eds. Handbook of Pain Assessment. New York: The Guilford Press; 2000;603-18.

33. Aaronson NK, Muller M, Cohen PD, et al. Translation, validation, and norming of the Dutch language version of the SF-36 Health Survey in community and chronic disease populations. J Clin Epidemiol 1998;51: 1055-68.

34. Ginkel JR, van Ark LA. SPSS syntax for missing value imputation in test and questionnaire data. Appl Psychol Meas 2005;29:152-3.

35. Twisk J, de Vente W. Attrition in longitudinal studies: how to deal with missing data. J Clin Epidemiol 2002;55:329-37.

36. Farrar JT, Young J, LaMoreaux L, et al. Clinical importance of changes in chronic pain intensity measured on an 11-point numerical pain rating scale. Pain 2001;94:149-58.

37. King M, Davidson O, Taylor F, et al. Effectiveness of teaching general practitioners skills in brief cognitive behaviour therapy to treat patients with depression: randomised controlled trial. BMJ 2002;324:947-50.

38. Jellema P, van der Windt DAWM, van der Horst HE, et al. Why is a treatment aimed at psychosocial factors not effective in patients with (sub)acute low back pain? Pain 2005;118:350-9. 